\title{
Pills, balloons or the knife: a review of the trials
}

\author{
A S Kurbaan, T J Bowker, A F Rickards
}

Atherosclerotic coronary artery disease is one of the leading causes of premature mortality and working life morbidity in industrialised nations. The medical objectives of the management of coronary disease are to maintain or improve the quality of life and to delay death. At any one point in the disease process patient management consists broadly of two options: medical treatment alone or medical treatment combined with revascularisation either by angioplasty (PTCA) or bypass surgery (CABG). Both strategies have shown benefits for patients, and both continue to change, generating a need for continual assessment of the role each contributes to patient management.

The factors involved in deciding on advice for a patient at any stage in the disease are numerous and complex, requiring access to as much information as possible on the state of disease. It is not reasonable to make a diagnosis of coronary artery disease or advise on anything but immediate management without the relevant information. "Blind" advice and treatment may appear to be economical and in the best traditions of conservative British medical practice but it is inappropriate for a physician to put a patient with coronary disease at risk because of failure to acquire or interpret easily available information.

Currently, there is reasonable information available to direct management strategies in patients presenting with stable angina. The data supporting strategies in acute syndromes (unstable angina and myocardial infarction) following the initial event is much less complete. Data supporting long term strategies where multiple interventions may be required at different times in the disease course, and selection of the order and type of intervention depending on the age of presentation and expected disease progression, is almost nonexistent.

\section{Management of patients with stable angina}

Angina is the main limiting symptom for patients with coronary artery disease. In no published trial does the pill do better than the balloon or the knife in relieving symptoms of angina.

In the early trials comparing surgery with medical treatment there was a consistent advantage for intervention in relieving angina in the short and medium term. This advantage may not be maintained in the long term, and survivors of both medical and surgical treatment experience a gradual recurrence of symptoms. In the Veterans Administration
Co-operative Study ${ }^{1}$ only $3 \%$ of the surgical group were free from angina at 15 years. Yusuf et $a l^{2}$ undertook a meta-analysis of three large and three smaller trials comparing medicine (1324 patients) with CABG (1325 patients). The 10 year mortality was $30 \%$ among the 1324 medically treated patients. The survival benefit in the CABG group was significant at five, seven, and 10 years, but the magnitude decreased over time (39\% at five years, $32 \%$ at seven years, and $17 \%$ at 10 years). By about 12 years there was a convergence of the survival curves. Immediate CABG was associated with significantly better outcome after five years if there was three vessel disease at baseline, but not if there was one or two vessel disease. Patients with single or double vessel disease had a mortality benefit after CABG that approached significance only in the presence of left anterior descending coronary artery stenosis. Hence, the trials showed an increasing benefit with CABG compared with medical therapy as the severity of coronary artery disease increased. The beneficial effect of CABG on left ventricular (LV) function, a constant finding, is probably similar in all patients, but is magnified in absolute terms by the much poorer prognosis of those with greater impairment of $\mathrm{LV}$ function.

The ACME trial ${ }^{3}$ compared PTCA with medical treatment for patients with single vessel disease and exercise induced myocardial ischaemia. Again the group undergoing intervention was more likely to be free from angina compared with the medically treated group, and at six months $64 \%$ of the medically treated group still had angina despite being on medication, compared with $46 \%$ of the angioplasty group who were largely not taking antianginal medication.

Pocock et $\mathrm{al}^{4}$ undertook a meta-analysis of nine randomised trials comparing intervention by PTCA and CABG in patients with angina. At one year of follow up the prevalence of angina, especially in those with multivessel disease, was greater in patients treated with PTCA compared with those treated with CABG. This advantage decreased with time probably because of a combination of repeat interventions in the PTCA group and early graft failure in the CABG group. The one year mortality was low with either treatment (CABG $2 \cdot 3 \% v$ PTCA $2.9 \%$ ).

Support for revascularisation for management of stable angina also comes from the observational Duke registry data which followed up 9263 patients with coronary disease confirmed by angiography between March 1984 and August 1990.5 They were graded according to the extent of coronary artery dis- 
ease, the dominant prognostic variable. Although the magnitude of the absolute survival differences was greatest for more severe coronary disease, there was a clear benefit from revascularisation in even the least severe disease. Furthermore, the registry indicated that the benefit:risk ratio favoured PTCA over CABG in less severe disease.

There is only one trial reporting with a three way comparison of medical treatment, PTCA, and CABG. In the Medicine, Angioplasty or Surgery Study (MASS) ${ }^{6}$ patients were selected with isolated proximal left anterior descending disease. At an average follow up of three years there was no difference in the mortality or infarction between the groups. No patient allocated to CABG needed further revascularisation compared with $10 \%$ and $11 \%$ of patients in the medical and angioplasty groups, respectively.

The Coronary Artery Surgery Study (CASS) ${ }^{7}$ gives us some insight into the natural history of coronary artery disease in terms of the future risk of myocardial infarction. In the CASS registry, the risk of infarction over three years was $2 \%$ for a stenosis of less than $50 \%$, $7 \%$ for a stenosis of $50-70 \%, 8 \%$ for a stenosis of $70-90 \%$, and $15 \%$ for a stenosis of $90-98 \%$. Although the risk of infarction was proportional to the severity of the stenosis in that vessel subtending "at risk" myocardium it must be appreciated that as the number of minor coronary lesions greatly outweigh the number of severe coronary lesions, some $80 \%$ of all myocardial infarctions during the follow up period occurred as a result of disease progression in vessels that had minor $(<50 \%$ stenosis) lesions.

Mortality in patients with coronary artery disease occurs as a result of myocardial infarction mediated either by catastrophic loss of myocardium, causing haemodynamic or electrical instability, or by the cumulative effects of muscle death, leading to intractable heart failure. If the majority of myocardial infarction in patients with coronary artery disease occurs in relation to haemodynamically insignificant lesions then one would not expect a revascularisation strategy aimed towards significant lesions to have a marked effect on subsequent survival. Logically one might predict that in patients at risk from catastrophic myocardial infarction (in the presence of multivessel coronary artery disease) revascularisation might have an impact on subsequent mortality, but in individuals with lesser degrees of disease where vessel occlusion might not produce overwhelming loss of myocardium, revascularisation would not necessarily confer a short to medium term survival benefit. From the available trial data this appears to be the case.

\section{Discussion}

It is important when interpreting the available data to bear in mind the progressive nature of coronary artery disease requiring further intervention, the continual development of the therapeutic modalities, and the changing patient population.
CROSSOVER BETWEEN DIFFERENT MODALITIES AND REPEAT REVASCULARISATION

In all published intervention trials there is considerable crossover between the modalities and need for further revascularisation as follow up is extended. In medical treatment versus CABG trials, crossover to CABG was $25 \%$ after five years, $33 \%$ after seven years, $40 \%$ after 10 years, and more than $50 \%$ after 15 years, on average. Patients with left main disease or three vessel disease were more likely to crossover than patients with one or two vessel disease. Interestingly, patients with impaired LV function were less likely to crossover. ${ }^{2}$

The need for second revascularisation after PTCA is recognised as one of the major limitations of the procedure. Less quoted is the reoperation rate in $\mathrm{CABG}$ groups. In the Veterans Administration Co-operative Study after 15 years, one third of patients had undergone repeat $\mathrm{CABG} .{ }^{1}$ Median time to repeat CABG was $9 \cdot 7$ years.

\section{CHANGING THERAPEUTIC MODALITIES}

Each of the three therapeutic modalities has continually changed compromising historical data from clinical trials. In the early medical treatment compared with CABG trials about two thirds of the patients were receiving $\beta$ blockers and about one fifth antiplatelet agents one year after randomisation in the medical group. This clearly is not representative of current management. The numbers taking lipid lowering agents were fewer still and the Scandinavian Simvastatin Survival Study ${ }^{8}$ suggests that the use of statins will lead to significant improvements in outcome-absolute risk reduction $3.3 \%$ and relative risk reduction $28.8 \%(P<0.001)$.

With the advent of stenting ${ }^{10}$ and the newer antiplatelet agents (GPIIB/IIIA blockers), ${ }^{11}$ the safety and efficacy of angioplasty techniques has improved.

Since the last patient was randomised into the CASS study in May 1979 surgical practice has improved. The increasing use of internal mammary grafts (only $9.9 \%$ of patients in the early trials) and routine antiplatelet therapy in the first year after CABG are likely to increase the patency rates of conduits, improving the benefits of CABG. More recently minimally invasive techniques have been developed, which may further improve the results. Analyses of the Duke registry ${ }^{10}$ tend to confirm that the survival offered by CABG may be improving.

\section{CHANGING PATIENT POPULATIONS}

Only relatively small numbers (5-10\%) of individuals screened were enrolled into the early trials, which tended to be dominated by younger male patients. In the meta-analysis of medicine versus CABG trials $96.8 \%$ of patients were men and only $7 \cdot 3 \%$ older than 60 years. $^{2}$ The data from registries such as CASS tends to suggest both females and older patients will derive benefit from revascularisation.

Increasing numbers of patients now present having had previous interventions and, in par- 
Time required to redeem the premium of revascularisation for different risk categories of patients and procedures. $T_{\text {high }}$ time taken to recover the penalty of an initial high risk intervention in a patient at high risk from disease; $T_{\text {low }}$ time taken to recover the penalty of an initial low risk intervention in a patient at low future risk from disease.

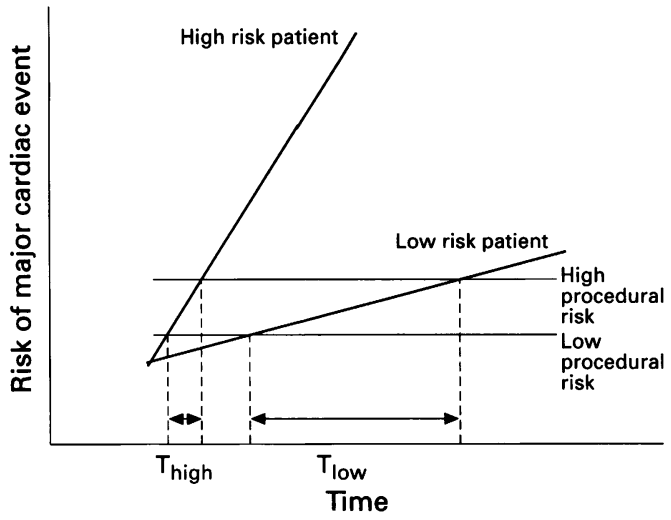

ticular, patients who have previous CABG are now presenting with graft failure. Although surgical and anaesthetic techniques have improved, the operative risk of repeat CABG remains double the risk of initial CABG. The operative mortality of repeat CABG may contribute to the increasing annual mortality rate in the surgical groups during late follow up. The optimal strategy in these patient remains unresolved but it is worth noting that patients who have had coronary surgery face an annual risk of death at around 10 years which is comparable to the initial presentation of unstable angina or three vessel disease and should justify aggressive management.

\section{BALANCING THE RISKS AND BENEFITS OF REVAS- CULARISATION}

Patients undergoing revascularisation procedures pay an immediate premium in terms of mortality and morbidity and the crucial issues are over what future period that premium is redeemed, and how that period relates to the life expectancy of the patient. The premium paid for revascularisation depends on the type and quality of the procedure, the age of the patient, and the state of disease. As operative techniques continue to improve the immediate premium has declined, however, patient age has increased making it more difficult to pay off the intervention premium as a reasonable proportion of the patient's future life. It may be unreasonable to advise an elderly patient with severe disease to undergo revascularisation unless quality of life can be improved. Equally, it might be reasonable to advise a younger asymptomatic patient with less threatening disease to pay an immediate operative penalty that will be recovered many times over in the future.

The figure tries to express this balancing equation graphically. A patient with high risk disease can pay off the premium of a high risk procedure over a short period of time. If the disease risk can be modified (such as by the use of statins) then a high risk procedure cannot be justified as the time to redeem the price of intervention may be too great a proportion of the patient's life expectancy. Thus, there is a complex relation between the age of the patient, the future risk of the disease, the effect of risk modification, and the risk of intervention, which has to be evaluated against the expected improvement in quality of life offered by PTCA or CABG.

\section{Conclusions}

There are enough data available from randomised trials and observational data to permit a physician confronted with a patient suffering from coronary disease to plan management. While not underestimating the complexity of decision making in patient management we suggest that the following guidelines should be kept in mind.

- Coronary artery disease is chronic, progressive, and incurable. It is potentially fatal but with a low overall mortality

- The prognosis of patients with coronary disease is largely dependent on the state of the coronary arteries, with prognosis worsening as disease increases

- As the age of the patient increases, prognosis may be more dependent on age and non-coronary disease rather than the state of coronary disease

- Death and infarction occur as a result of coronary occlusion, which may result from a lesion of any severity, although the risk of occlusion increases as lesion severity increases

- Revascularisation should be directed to those patients with severe lesions where symptoms are likely to be present and better managed by revascularisation or where the consequences of occlusion are likely to be catastrophic

- The risk of intervention must be balanced against risk of non-intervention and the symptomatic benefit of intervention. All intervention carries a mortality premium that must be paid off over a reasonable time where "reasonable" is related to expected survival, which, in turn, is largely determined by the age of the patient

- Medical management should be directed to all patients (with severe and less severe disease) to reduce the chances of lesion progression and subsequent coronary occlusion, even though the effects may not be judged to be catastrophic.

Pills, balloons or the knife? The answer is, all three judiciously used and planned by an intelligent physician with a clear understanding of the patient's age, clinical state, coronary anatomy, and expected survival.

1 The VA Coronary Artery Bypass Surgery Cooperative Study Group. Eighteen year follow-up in the Veteran Affairs Cooperative Study of coronary bypass surgery fo stable angina. Circulation 1992;86:121-30.

2 Yusuf S, Zucker D, Peduzzi P, Fisher LD, Takaro T, Ward Kennedy J, et al. Effect of coronary artery bypass graft surgery on survival: overview of 10 year results from ran domised trials by the Coronary Artery Bypass Graf Surgery Trialists Collaboration. Lancet 1994;344: 563-70

3 Parisi AF, Folland ED, Hartigan $P$ (on behalf of the Veterans Affairs ACME Investigators). A comparison of angioplasty with medical therapy in the treatment of single vessel coronary artery disease. $N$ Engl f Med 1992;326: $10-16$.

4 Pocock SJ, Henderson RA, Rickards AF, Hampton JR, King III SB, Hamm CW, et al. Meta-analysis of randomised trials comparing coronary angioplasty with bypass surgery. Lancet 1995;346:1 184-9.

5 Jones RH, Kesler K, Phillips III HR, Mark DB, Smith PK, Nelson CL, et al. Long-term survival benefits of coronary Nelson CL, et al. Long-term survival benefits of coronary artery bypass grafting and percutaneous transluminal Thorac Cardiovasc Surg 1996;111:1013-125.

6 Heub W, Bellotti G, Almeida S, de Oliveira SA, Arie S, de Albuquerque CP, et al. The Medicine, Angioplasty or 
Surgery Study (MASS): a prospective randomised trial for single proximal left anterior descending artery

7 Ellis S, Alderman E, Cain K, Fisher L, Sanders W Bourassa M, CASS Investigators. Prediction of risk of anterior myocardial infarction by lesion severity and measurement method of stenoses in the left anterior descending distribution: a CASS Registry study. $f \mathrm{Am}$ Coll Cardiol 1988;11:908-16.

8 Scandinavian Simvastatin Survival Study Group. Randomised trial of cholesterol lowering in 4444 patients with coronary disease: the Scandinavian Simvastatin with coronary disease: the Scandinavian S
Survival Study (4S). Lancet 1994;344:1383-9.
9 Califf RM, Harrell FE, Lee KL, Scott Rankin J, Hlatky MA, Mark DB, et al. The evolution of medical and surgical therapy for coronary artery disease: A 15 year perspective. FAMA 1989;261:2077-86.

10 Serruys $P W$, de Jaegere $P$, Kiemeneiji C, Macaya $H$, Rutsch W, Heyndrickx G, et al. A comparison of balloon expandable stent implantation with balloon angioplasty in patients with coronary artery disease. (The Benestent study.) N Engl ₹ Med 1994;331:489-95.

11 The EPIC investigators. Use of a monoclonal antibody directed against the platelet glycoprotein IIb/IIIa receptor in high risk coronary angioplasty. N Engl $₹$ Med 1994; 330:1031-7.

\title{
What the randomised trials don't tell us about the shortcomings of CABG
}

\author{
Stephen Westaby
}

The vintage trials of medical versus surgical (CABG) treatment for coronary artery disease showed early CABG to prolong life for patients with left main stem and severe three vessel disease. In Europe, where surgical intervention rates are less than 500/million population, operation is best reserved for patients with three vessel disease, particularly when left ventricular function is impaired. In this context, debate between the merits of angioplasty (PTCA) and CABG is fallacious and the trials provide no information about the value of sequential intervention. Since beginning RITA (Randomised intervention treatment of angina) and others there has been a sea change in drug treatment and catheter intervention. Intracoronary stents and improved moderation of risk factors now convey important benefits.

The aims of both modes of treatment are to relieve symptoms and prolong life by preventing myocardial infarction. For many patients the combination of modern drug treatment and PTCA achieves this goal without detracting from quality of life. There are two broad categories of patient with coronary disease. First, those with genetic predisposition or diabetes present between 40 and 65 years of age with aggressive pathology and will spend the rest of their lives battling symptoms and events. These patients require constant supervision even during symptom free periods. A strategy of PTCA first and surgery in time may convey prognostic benefit compared with early CABG. Arguably, bypass conduits should be preserved for a critical stage in the disease process and arterial grafts (internal mammary, radial, and gastroepiploeic arteries) used preferentially in the first instance. Venous conduits have a limited life span and reoperation in the presence of three severely atheromatous vein grafts carries an operative mortality up to $30 \%$. For the second group, those who present after age 65 , a careful operation with a left internal mammary artery (LIMA) to the left anterior descending (LAD) coronary artery and appropriate good quality vein grafts may prove definitive. However, surgical waiting lists (up to 18 months in the UK) result in substantial mortal- ity $(5 \%)$, and risk of intervening myocardial infarction. PTCA contributes by keeping single or two vessel disease away from the surgeon and by addressing a culprit lesion in three vessel disease pending surgery.

Given the choice, most patients prefer one or more PTCA to conventional CABG. While there is little difference in hospital mortality, surgical morbidity enters into the equation. The damaging effects of cardiopulmonary bypass cause subtle but prolonged neuropsychological effects in $50-60 \%$ of patients and $2-3 \%$ suffer a stroke. In the randomised trials perioperative myocardial infarction occurred in $8-10 \%$ of CABG patients (versus $2 \%$ PTCA) and between $5 \%$ and $10 \%$ suffered sternal or leg wound problems. PTCA carries less morbidity although the risks of coronary occlusion followed by emergency surgery are significant. PTCA is less satisfactory for diabetics, where comparative five year mortality is $35 \%$ versus $19 \%$ for CABG.

With an increasingly elderly population, economic constraints will shape future strategy and reinforce the drive towards catheter intervention. In a society where heart failure consumes enormous financial resources, a coordinated effort between cardiologists and surgeons is required to prevent myocardial infarction and preserve left ventricular function. To this end, screening should be considered for high risk groups.

The role of less invasive CABG (limited thoracotomy, no cardiopulmonary bypass) is yet to be defined. This may prove worthwhile for a culprit lesion in the LAD or left main stem in patients at increased risk from conventional surgery (those with renal failure, chronic obstructive airways disease, and some reoperations). A patent LIMA graft to the LAD has important prognostic implications. A combination of minimally invasive LIMA to LAD with PTCA to other vessels may prove helpful in the frail, elderly patient, while transmyocardial revascularisation appears to provide symptomatic relief in "inoperable" disease. The prognostic value of sequential PTCA then CABG remains to be seen. 